\title{
Usefulness of phalangeal quantitative ultrasound in identifying reduced bone mineral status and increased fracture risk in adolescents with Turner syndrome
}

\author{
Francesco Vierucci, ${ }^{1}$ Marta Del Pistoia, ${ }^{1}$ Paola Erba, ${ }^{2}$ \\ Giovanni Federico, ${ }^{1}$ Giuseppe Saggese ${ }^{1}$ \\ ${ }^{1}$ U.O. Pediatria Universitaria, Dipartimento Materno-Infantile, ${ }^{2}$ U.O. Medicina Nucleare, Dipartimento di Scienze \\ Oncologiche e Radiologiche; Azienda Ospedaliero-Universitaria Pisana; Pisa, Italy
}

\begin{abstract}
OBJECTIVE. Bone health is a major concern in patients with Turner syndrome (TS). There are few studies assessing bone mineral status in TS adolescents and none have reported a clear relationship with the risk of fracture. We assessed bone mineral status at three different skeletal sites by two different densitometric techniques in a group of TS adolescents. DESIGN. In 24 TS adolescents $(17.1 \pm 3.1$ years) we evaluated lumbar and femoral volumetric bone mineral density (vBMD) with dual energy X-ray absorptiometry (DXA), amplitude-dependent speed of sound (AD-SoS) and bone transmission time (BTT) with phalangeal quantitative ultrasound (QUS). RESULTS. Mean lumbar vBMD Z-score was normal, while mean femoral vBMD, ADSoS and BTT Z-score were reduced. 8/24 (33.3\%) and 13/24 (54.2\%) girls had AD-SoS and BTT $\leq-2 \mathrm{Z}$-score, respectively, while lumbar vBMD and femoral vBMD were $\leq-2 \mathrm{Z}$-score only in $2 / 24$ (8.4\%) and 1/24 (4.2\%) patients. Overall, we documented 15 fractures (three pathological) in 8 girls. Patients who reported at least one fracture had lower AD-SoS and BTT Z-score values than fracture-free girls. The presence of a value of BTT $\leq-2.0 \mathrm{Z}$-score was associated with a significant $O R$ of positive history of fracture of $11.67\left(\chi^{2}=5.906, p=0.015\right.$, C.I. 95\% 1.14-119.54). Lumbar and femoral vBMD were not related to fracture risk. CONCLUSIONS. TS adolescents may have impaired bone mineral status in skeletal sites with predominant cortical bone. Phalangeal QUS represents a useful method to identify subjects with increased fracture risk.

Key words: Bone mineral status, Dual energy X-ray absorptiometry, Quantitative ultrasound, Turner syndrome
\end{abstract}

Address for correspondence:

Francesco Vierucci, MD, U.O. Pediatria Universitaria, Dipartimento Materno-Infantile, Azienda OspedalieroUniversitaria Pisana, Via Roma 67, 56126 Pisa, Italy, Tel./Fax: +39050 993109, E-mail: vieruf@hotmail.it Received 10-6-2013, Accepted 24-9-2013

\section{INTRODUCTION}

Turner syndrome (TS) is a complex condition associated with skeletal involvement. ${ }^{1,2}$ Most studies using dual-energy X-ray absorptiometry (DXA) found decreased bone mineral density (BMD)., Several 
factors as hypogonadism, ${ }^{5}$ chromosomal alterations (mosaicism) ${ }^{6}$ and Short stature HOmeoboX (SHOX)related haploinsufficiency disorders ${ }^{7,8}$ can affect bone mass and geometry in TS girls. Recent studies have confirmed an intrinsic oestrogen-independent defect of bone mass and geometry mainly in skeletal sites with a higher content of cortical bone, such as the femoral neck and long bones. ${ }^{9-12}$ Conversely, it was observed that TS patients receiving appropriate oestrogen treatment usually showed normal trabecular BMD after adjusting for body size. ${ }^{13-17}$ In addition to Hormonal Replacement Therapy (HRT), Growth Hormone $(\mathrm{GH})$ administration is considered standard treatment for TS girls, but the role of GH in promoting bone accrual has not yet been completely defined..$^{18-20}$

Several studies reported in TS patients an increase in fracture prevalence and risk that was higher during childhood and after the age of 45.,21 Fractures occurred mainly in skeletal sites consisting predominantly of cortical bone, often after a high or medium force trauma. ${ }^{22,23}$ However, the most recently published guidelines for the care of girls and women with TS do not recommend BMD assessment during childhood and/or adolescence. ${ }^{24}$ It must be stressed that there is, as yet, no definition of when the defect in bone mass acquisition starts and whether early recognition could improve bone health. Quantitative ultrasound (QUS) is a densitometric technique of particular interest during paediatric age because it is easy to perform, non-invasive and radiation-free. At present, data on bone mineral status in TS girls assessed by QUS are lacking.

The aim of this study was to evaluate bone mineral status in adolescent girls with TS by two different densitometric techniques, DXA and phalangeal QUS, and to assess whether a reduced bone status was related to the history of fracture.

\section{MATERIALS AND METHODS}

\section{Patients}

Twenty-four Caucasian Italian girls with TS aged 17.1 \pm 3.1 years (range 8.7-19.9) were referred as outpatients to the Division of Paediatric Endocrinology of the University of Pisa, Italy. The diagnosis of TS was made by karyotyping ( $45 \mathrm{X} n=13$; deletion $n=3$; mosaic $n=8)$. Clinical details are summarized in Table 1 .
Table 1. Clinical findings in TS girls and in controls

\begin{tabular}{lcc}
\hline Turner syndrome girls & n & Mean \pm SD (range) \\
\hline Age at diagnosis, years & 24 & $8.9 \pm 3.1(0.1-14.1)$ \\
Age at start of GH therapy, years & 24 & $9.3 \pm 2.9(2.0-14.2)$ \\
Age at start of HRT, years & 21 & $13.4 \pm 0.8(12.0-15.0)$ \\
Duration of GH therapy, years & $24^{*}$ & $6.9 \pm 2.8(2.4-12.0)$ \\
Duration of HRT, years & 21 & $4.6 \pm 1.9(0.9-7.2)$ \\
Stature, Z-score & 24 & $-1.8 \pm 0.8(-3.3 ;-0.3)$ \\
BMI, Z-score & 24 & $0.9 \pm 0.9(-0.6 ; 3.2)$ \\
\hline
\end{tabular}

* 15 girls had stopped GH therapy from $1.4 \pm 0.7$ years (range $0.1-2.5)$

GH: growth hormone; HRT: hormone replacement therapy; BMI: body mass index.

None of the patients was affected by any other disease known to be associated with abnormal bone mineral status. Two patients, affected by autoimmune thyroiditis, had normal blood levels of thyroid hormones. There were no contraindications to patients taking part in non-competitive sport activities. $\mathrm{Hu}-$ man recombinant $\mathrm{GH}$ was administered at a dosage of $0.330-0.375 \mathrm{mg} / \mathrm{Kg} /$ week. ${ }^{24}$ Pubertal development was induced in 21 patients with conjugate oestrogens at a dosage of $0.3 \mathrm{mg}$ daily for 6 months, followed by $0.625 \mathrm{mg}$ daily. From the second year, cyclic HRT was started (conjugate oestrogens $0.625 \mathrm{mg}$ daily for 21 days, adding progesterone for 10 days/cycle). The remaining three girls were too young to induce puberty.

\section{Study design}

In all the girls we evaluated lumbar and femoral BMD by DXA and phalangeal amplitude-dependent speed of sound (AD-SoS) and bone transmission time (BTT) by QUS to assess bone mineral status.

Lumbar and femoral BMD of patients were compared with appropriate age-reference values for the girls, which were obtained with the same DXA apparatus and software. ${ }^{25,26}$ Phalangeal AD-SoS and BTT results were compared with our own reference data for QUS. ${ }^{27}$

Standing height and body weight of all the patients were measured with a wall-mounted stadiometer and a mechanical balance. In each patient, both length and weight were the mean of three measurements. Body Mass Index (BMI) was calculated using the formula weight $(\mathrm{Kg}) /$ height $(\mathrm{m})^{2}$. Height, weight and 
BMI were expressed as Z-score according to the LMS method of Cole et al. ${ }^{28}$

Written informed consent to the study for all the enrolled girls was obtained from parents or from the girls as requested. The study was approved by the Ethics Committee for Human Investigation at our Institution.

\section{Assessment of bone mineral status and prevalence of fracture}

Lumbar and femoral areal BMD (aBMD) were measured by postero-anterior DXA (Lunar DPX-L, Lunar Radiation Corp., Madison, WI, USA) at both the lumbar spine (L2-L4) and femoral neck. Since TS phenotype is characterized by short stature ${ }^{13}$ and DXA is a two-dimensional technique influenced by bone dimensions, smaller bones have lower aBMD. ${ }^{29}$ Thus we calculated lumbar and femoral BMDvolume (vBMD) according to Kroger et $\mathrm{al}^{26}$ to reduce the confounding effect of the small vertebrae and femoral neck of TS girls in BMD assessment. The coefficient of precision, in vivo, was less than $1.0 \%$ for both lumbar aBMD and vBMD, and $2.3 \%$ for femoral aBMD and vBMD. ${ }^{30}$

QUS (DBM Sonic, IGEA, Carpi, Modena, Italy) was carried out as previously described $.^{27} \mathrm{AD}-\mathrm{SoS}$ is a QUS variable reflecting the speed of sound $(\mathrm{m} / \mathrm{s})$ through the proximal phalanx. ${ }^{31}$ BTT ( $\mu$ s) derives from the difference between the time when the first peak of the signal received reaches its maximum and the time that would be measured if no bone but only soft tissue were present between the transducers. Thus, BTT is largely independent of ultrasound attenuation and soft tissue bias and it depends only on bone properties. ${ }^{32}$ The final result is the average AD-SoS and BTT over four fingers (digit II - V). All the QUS measurements were carried out by the same operator on the non-dominant hand. The coefficients of precision, in vivo, were $0.8 \%$ and $1.5 \%$ for $\mathrm{AD}-\mathrm{SoS}$ and BTT, respectively. ${ }^{27}$

Examination of non-dominant wrist and hand $\mathrm{X}$-rays routinely performed in TS girls allowed us to exclude the presence of phalangeal bone deformities that could influence QUS measurement.

Low bone mineral status was defined in the presence of at least one densitometric parameter $\leq-2.0$
Z-score among lumbar vBMD, femoral vBMD, ADSoS or BTT.

For all patients, frequency, severity of injury and sites of fractures were recorded by a questionnaire. The sites of fractures were confirmed by examining the plain radiographs and/or the X-ray medical reports. We classified fractures as spontaneous (no recognizable trauma) or traumatic according to the Landin severity scale. ${ }^{33}$ Multiple fractures of the small bones of the hand or foot were recorded as one fracture regardless of the number of fractured bones. Fracture prevalence was defined as the percentage of patients who had at least one fracture from birth to the time of assessment.

\section{Statistical analysis}

The results are expressed as mean $\pm \mathrm{SD}$. Comparison of mean Z-scores of densitometric variables with the hypothetical mean of 0.0 was performed by one sample $t$ test. Comparison of auxological and densitometric data according to the history of fracture was assessed by the non-parametric Mann-Whitney test. Fisher's exact test was used to compare groups of categorical variables. Odds ratios (OR) between bone mineral status and history of fracture were evaluated by the chi-square test. Spearman correlation coefficients were adopted to explore the relationship between densitometric variables and age at diagnosis, age at start of HRT or GH therapy, duration of HRT or GH therapy. All statistical analyses were carried out using the SPSS (Statistical Package of Social Sciences, Chicago, IL, USA) for Windows software program version 19.0. A p value $<0.05$ was considered significant.

\section{RESULTS}

Lumbar aBMD, femoral aBMD and vBMD Zscores were significantly reduced in comparison with the hypothetical mean of $0.0(-1.2 \pm 1.1, \mathrm{p}<0.001$; $-1.4 \pm 0.9, \mathrm{p}<0.001 ;-0.7 \pm 1.0, \mathrm{p}=0.001$, respectively), while mean lumbar vBMD Z-score was normal $(-0.3 \pm 0.9, \mathrm{p}=0.105)$. AD-SoS and BTT Z-scores were significantly reduced $(-1.4 \pm 1.2, \mathrm{p}<0.001 ;-1.8 \pm 1.1$, $\mathrm{p}<0.001$, respectively).

Overall, 14/24 (58.3\%) girls had low bone mineral status. Lumbar BMD, femoral BMD, AD-SoS and 
BTT were $\leq-2$ Z-score in 2/24 (8.4\%), 1/24 (4.2\%), $8 / 24(33.3 \%)$ and $13 / 24(54.2 \%)$ girls, respectively.

Eight patients with TS had at least one fracture (prevalence of fracture 33.3\%). In particular, 3 patients reported 1 fracture, 4 patients 2 fractures and 1 patient 4 fractures. Overall, we documented 15 fractures, 3 of which were spontaneous (1 patient fractured her right tibial distal metaphysis while walking; 1 patient fractured her right foot twice, the first time while she was climbing stairs and the second while walking). All fractures involved the appendicular skeleton (radial or tibial distal metaphysis), but not vertebral or femoral sites.

We did not find significant differences between fractured and fracture-free patients as regards auxological parameters, age at start and duration of $\mathrm{GH}$ therapy and HRT (Table 2).

DXA parameters did not differ between the two groups and resulted in the normal range for age and sex-matched reference data in 7/8 (87.5\%) girls with a positive history of fracture. However, QUS parameters were significantly lower in patients who had at least one fracture than in fracture-free patients.

In girls with a positive history of fracture, AD-
SoS and BTT were $\leq-2.0 \mathrm{Z}$-score in 5/8 (62.5\%) and $7 / 8$ (87.5\%), respectively. Only one TS girl with a positive history of fracture showed a normal QUS. In particular, the two patients with a history of spontaneous fractures showed values of both $\mathrm{AD}-\mathrm{SoS}$ and BTT Z-score $\leq-2.0$ while having lumbar and femoral vBMD values in the normal range.

In TS girls with a negative history of fracture, $\mathrm{AD}$ SoS and BTT were $\leq-2.0 \mathrm{Z}$-score in 3/16 (18.8\%; $\mathrm{p}=$ 0.065 vs fractured girls) and $6 / 16(37.5 \%, \mathrm{p}=0.036$ vs fractured girls), respectively.

The presence of a value of BTT $\leq-2.0 \mathrm{Z}$-score was associated with a significant OR of positive history of fracture of $11.67\left(\chi^{2}=5.906, p=0.015\right.$, C.I. $95 \%$ 1.14-119.54). In contrast, DXA variables were not associated with history of fracture (data not shown).

Bone mineral status did not differ in relation to karyotype (data not shown). Densitometric parameters did not correlate with age at diagnosis, age at start of HRT or GH therapy, duration of HRT or $\mathrm{GH}$ therapy (data not shown).

\section{DISCUSSION}

Bone health is a major concern for women with

Table 2. Anthropometric and densitometric evaluation according to the history of fractures

\begin{tabular}{lccc}
\hline & Unfractured $(\mathbf{n}=\mathbf{1 6})$ & Fractured $(\mathbf{n}=\mathbf{8})$ & p \\
& Mean \pm SD & $9 \pm 2.5$ & 0.878 \\
\hline Age at diagnosis, years & $8.8 \pm 3.4$ & $9.3 \pm 2.5$ & 0.759 \\
Age at start GH therapy, years & $9.2 \pm 3.1$ & $13.1 \pm 0.7$ & 0.363 \\
Age at start HRT, years & $13.5 \pm 0.9(\mathrm{n}=13)$ & $7.8 \pm 2.7^{* *}$ & 0.298 \\
Duration of GH therapy, years & $6.5 \pm 2.8^{*}$ & $5.3 \pm 1.7$ & 0.103 \\
Duration of HRT, years & $+1.1 \pm 1.9(\mathrm{n}=13)$ & $17.3 \pm 2.3$ & 0.927 \\
Age at densitometric evaluation, years & $16.9 \pm 3.5$ & $-1.9 \pm 0.6$ & 0.539 \\
Stature at densitometric evaluation, Z-score & $-1.7 \pm 0.9$ & $1.1 \pm 0.9$ & 0.623 \\
BMI at densitometric evaluation, Z-score & $0.8 \pm 0.9$ & $-1.5 \pm 1.1$ & 0.408 \\
Lumbar aBMD, Z-score & $-1.1 \pm 1.1$ & $-0.3 \pm 1$ & 0.951 \\
Lumbar vBMD, Z-score & $-0.3 \pm 0.9$ & $-1.8 \pm 1.3$ & 0.230 \\
Femoral aBMD, Z-score & $-1.3 \pm 0.5$ & $-0.8 \pm 1.6$ & 0.644 \\
Femoral vBMD, Z-score & $-0.7 \pm 0.7$ & $-2.1 \pm 0.6$ & 0.021 \\
AD-SoS, Z-score & $-1.1 \pm 1.3$ & $-2.4 \pm 0.5$ & 0.023 \\
BTT, Z-score & $-1.4 \pm 1.1$ & & \\
\hline
\end{tabular}

* 8 girls had stopped GH therapy from $1.3 \pm 0.6$ years (range $0.1-2.1$ ).

** 7 girls had stopped GH therapy from $1.6 \pm 0.8$ years (range $0.6-2.5$ ). 
TS. ${ }^{2-4,34}$ This study assessed bone mineral status in a group of adolescents with TS at three different skeletal sites by two different densitometric techniques. Our results confirmed that TS girls had an impaired bone mineral status in skeletal sites with predominant cortical bone such as femoral neck and phalanges. Moreover, TS adolescents with BTT $\leq-2.0$ Z-score had a 12-fold increased OR of positive history of fracture, highlighting the importance of QUS evaluation in TS during adolescence.

TS patients have a slightly increased risk of fractures than subjects with normal karyotype, particularly during childhood and after the age of $45 .^{4,21}$ Fractures are generally caused by high or medium force trauma and involve the appendicular skeleton which consists predominantly of cortical bone. ${ }^{22}$ The assessment of fracture prevalence in TS subjects is based mainly on retrospective studies, most of which were carried out on a mixed population of children, adolescents and adults. Available studies reported a wide range of fracture prevalence, ranging from $10 \%$ to more than $50 \% .{ }^{3,4,9,10,14,21,23,35-38}$ Only three studies enrolled exclusively children and adolescents and in two the prevalence of fracture was $21 \%{ }^{10}$ and $27 \%,{ }^{9}$ a figure similar to our own results. Interestingly, both the studies reported a reduced cortical vBMD at the proximal radius measured by peripheral Quantitative Computed Tomography (pQCT). In particular, Soucek and colleagues ${ }^{10}$ found that girls with a positive history of fractures had a lower total vBMD at the radial metaphysis compared with non-fractured TS girls. This finding is in agreement with our own results that showed a lower phalangeal bone mineral status by QUS in girls having a positive history of fracture.

We confirmed that adolescents with TS had a normal lumbar vBMD and a reduced vBMD at the femoral neck. Previous studies showed that GH- and HRT-treated patients had normal lumbar BMD by DXA, suggesting that the axial trabecular bone was preserved in young adults with TS., ${ }^{913,15}$

On the other hand, skeletal sites with predominantly cortical bone had a reduced BMD measured by DXA, pQCT and QUS. ${ }^{9-12,38,39}$ Recently, Hansen and colleagues, using high-resolution pQCT, observed that adult TS women (mean age 35 years, range 20 61) also had defective trabecular micro-architecture at the distal radius and tibia compared to healthy adults. ${ }^{40}$ Since proximal phalanges of the hands contain both cortical $(\sim 60 \%)$ and trabecular bone $(\sim 40 \%)$, phalangeal QUS evaluation appears of considerable benefit as it gives integrate information on bone health in TS girls. ${ }^{41}$ The present study is the first using phalangeal QUS to assess bone mineral status in these patients. This technique has the advantage of measuring BTT that reflects the bone properties independently of the confounding effect of soft tissues. ${ }^{41}$ This aspect is particularly useful in girls with TS, who often have an excess of adipose tissue that could affect densitometric assessment. ${ }^{32}$ In addition, QUS measurement also provides information on bone architecture, elasticity and particularly bone quality. ${ }^{41}$ Our results are in agreement with the study of Zuckerman-Levin and colleagues performed in 27 girls and women with TS (age range 11-39 years). Using QUS, the authors found a reduced radial and tibial SoS Z-score in comparison with control subjects matched for age or height, due to a selective defect in cortical bone in TS. ${ }^{38}$

One important question is at what age bone mineral status starts to be affected in TS patients. Our observation that phalangeal QUS was reduced during adolescence suggests that factors affecting bone status were already operative at a younger age, possibly before puberty. Recently, Soucek and colleagues performed pQCT of the forearm in 24 prepubertal TS girls (mean age 10.3 years) and in 10 prepubertal children with isolated SHOX deficiency. Both groups had similar alteration in bone geometry (increased total bone area, decreased relative cortical bone area and thin cortex at the proximal radius) with normal radial bone strength, confirming that an intrinsic oestrogen-independent genetic alteration (loss of one copy of SHOX) causes the particular bone phenotype of TS, already present in prepubertal age. ${ }^{8}$ Prospective studies are needed to evaluate whether this altered bone geometry may determine an increase in fracture risk after puberty and in adulthood.

Having in mind the above observation and considering that: a) QUS is an easy to use, radiation-free and portable technique particularly appropriate during paediatric age and for long-term follow-up; ${ }^{42-45} \mathrm{~b}$ ) our findings show that phalangeal QUS may be useful as pQCT in assessing bone health in children and ado- 
lescents with TS; c) we and others found that fractures most frequently occurred during the peripubertal period. ${ }^{9}{ }^{10}$ We thus recommend that bone mineral status be routinely assessed by phalangeal QUS in TS girls during the pediatric age, starting before the onset of puberty. The identification of TS patients with a low bone mineral status may help in correcting modifiable risk factors such as vitamin D status, calcium intake and physical activity to improve bone health. The presence of two girls in our series who suffered from spontaneous fractures, a finding reported by another paediatric series,${ }^{10}$ highlights the importance of early detection of patients at increased risk of fractures.

Similarly to our results, other recent paediatric studies reported an association between phalangeal QUS variables and bone health. A recent study conducted in 1,719 children and adolescents with bone disorders showed that phalangeal QUS was able to identify fractured patients, reflecting the severity of the causative trauma with a high discrimination power for fragility fractures. ${ }^{46}$ Mussa and colleagues reported that a BTT decreasing more than 1 Standard Deviation over 6 months was able to predict skeletal complication occurrence in children treated for acute lymphoblastic leukemia. ${ }^{44}$

Our study presents some limitations. The number of TS girls enrolled in the present study was small, but comparable with the population examined in previous studies performed in paediatric subjects. At the time of evaluation the patients had been on $\mathrm{GH}$ administration and HRT for a variable period of time, possibly affecting the correlations between bone mineral status and treatments. SHOX haploinsufficiency may affect skeletal growth, thus TS girls differ in body size from normal subjects, perhaps influencing the comparison of densitometric evaluation with reference data. Finally, we performed a retrospective study, thus we were unable to assess other factors that could influence bone mass accrual such as physical activity, vitamin D status and dietary calcium intake.

In conclusion, we found that TS adolescents may have impaired bone mineral status. Since TS patients with BTT $\leq-2$ Z-score had a significantly increased OR of fracture, it seems reasonable to assess bone mineral status in TS during paediatric age by phalan- geal QUS for early detection of subjects at increased risk of fracture. Prospective studies on larger paediatric series are needed to confirm our results and to study new strategies and treatments to preserve bone health in TS.

\section{DISCLOSURE SUMMARY}

The authors have nothing to disclose.

\section{REFERENCES}

1. Turner HH, 1938 A syndrome of infantilism, congenital webbed neck, and cubitus valgus. Endocrinology 23: 566-574.

2. Shore RM, Chesney RW, Mazess RB, Rose PG, Bargman GJ, 1982 Skeletal demineralization in Turner's syndrome. Calcif Tissue Int 34: 519-522.

3. Davies MC, Gulekli B, Jacobs HS, 1995 Osteoporosis in Turner's syndrome and other forms of primary amenorrhoea. Clin Endocrinol 43: 741-746.

4. Landin-Wilhelmsen K, Bryman I, Windh M, Wilhelmsen L, 1999 Osteoporosis and fractures in Turner syndrome: importance of growth promoting and oestrogen therapy. Clin Endocrinol 51: 497-502.

5. Pitukcheewanont P, Numbenjapon N, Safani D, Rossmiller S, Gilsanz V, Costin G, 2011 Bone size and density measurements in prepubertal children with Turner syndrome prior to growth hormone therapy. Osteoporos Int 22: 1709-1715.

6. El-Mansoury M, Barrenäs ML, Bryman I, et al, 2007 Chromosomal mosaicism mitigates stigmata and cardiovascular risk factors in Turner syndrome. Clin Endocrinol (Oxf) 66: 744-751.

7. Oliveira CS, Alves C, 2011 The role of the SHOX gene in the pathophysiology of Turner syndrome. Endocrinol Nutr 58: 433-442.

8. Soucek O, Zapletalova J, Zemkova D, et al, 2013 Prepubertal girls with Turner syndrome and children wth isolated SHOX deficiency have similar bone geometry at the radius. J Clin Endocrinol Metab 98: E1241-1247.

9. Holroyd CR, Davies JH, Taylor P, et al, 2010 Reduced cortical bone density with normal trabecular bone density in girls with Turner syndrome. Osteoporos Int 21: 2093-2099.

10. Soucek O, Lebl J, Snajderova M, et al, 2011 Bone geometry and volumetric bone mineral density in girls with Turner syndrome of different pubertal stages. Clin Endocrinol (Oxf) 74: 445-452.

11. Bechtold S, Rauch F, Noelle V, et al, 2001 Musculoskeletal analyses of the forearm in young women with Turner syndrome: a study using peripheral quantitative computed tomography. J Clin Endocrinol Metab 86: 5819-5823.

12. Mora S, Weber G, Guarneri MP, Nizzoli G, Pasolini 
D, Chiumello G, 1992 Effect of estrogen replacement therapy on bone mineral content in girls with Turner syndrome. Obstet Gynecol 79: 747-751.

13. Bertelloni S, Cinquanta L, Baroncelli GI, Simi P, Rossi S, Saggese G, 2000 Volumetric bone mineral density in young women with Turner's syndrome treated with estrogens or estrogens plus growth hormone. Horm Res 53: $72-76$.

14. Bakalov VK, Chen ML, Baron J, et al, 2003 Bone mineral density and fractures in Turner syndrome. Am J Med 115: 259-264.

15. Shaw NJ, Rehan VK, Husain S, Marshall T, Smith CS, 1997 Bone mineral density in Turner's syndrome - a longitudinal study. Clin Endocrinol (Oxf) 47: 367-370.

16. Kodama M, Komura H, Kodama T, Nishio Y, Kimura T, 2012 Estrogen therapy initiated at an early age increases bone mineral density in Turner syndrome patients. Endocr J 59: 153-159.

17. Högler W, Briody J, Moore B, Garnett S, Lu PW, Cowell CT, 2004 Importance of estrogen on bone health in Turner syndrome: a cross-sectional and longitudinal study using dual-energy X-ray absorptiometry. J Clin Endocrinol Metab 89: 193-199.

18. Bakalov VK, Van PL, Baron J, Reynolds JC, Bondy CA, 2004 Growth hormone therapy and bone mineral density in Turner syndrome. J Clin Endocrinol Metab 89: 4886-4889.

19. Aycan Z, Cetinkaya E, Darendeliler F, et al, 2008 The effect of growth hormone treatment on bone mineral density in prepubertal girls with Turner syndrome: a multicentre prospective clinical trial. Clin Endocrinol (Oxf) 68: 769-772.

20. Ari M, Bakalov VK, Hill S, Bondy CA, 2006 The effects of growth hormone treatment on bone mineral density and body composition in girls with Turner syndrome. $\mathrm{J}$ Clin Endocrinol Metab 91: 4302-4305.

21. Ross JL, Long LM, Feuillan P, Cassorla F, Cutler GB Jr, 1991 Normal bone density of the wrist and spine and increased wrist fractures in girls with Turner's syndrome. J Clin Endocrinol Metab 73: 355-359.

22. Bakalov VK, Bondy CA, 2008 Fracture risk and bone mineral density in Turner syndrome. Rev Endocr Metab Disord 9: 145-151.

23. Gravholt CH, Juul S, Naeraa RW, Hansen J, 1998 Morbidity in Turner syndrome. J Clin Epidemiol 51: 147-158.

24. Bondy CA; Turner Syndrome Study Group, 2007 Care of girls and women with Turner syndrome: a guideline of the Turner Syndrome Study Group. J Clin Endocrinol Metab 92: 10-25.

25. Boot AM, de Ridder MA, Pols HA, Krenning EP, de Muinck Keizer-Schrama SM, 1997 Bone mineral density in children and adolescents: relation to puberty, calcium intake, and physical activity. J Clin Endocrinol Metab 82: 57-62.

26. Kroger H, Kotaniemi A, Vainio P, Alhava E, 1992 Bone densitometry of the spine and femur in children by dual- energy X-ray absorptiometry. Bone Miner 17: 75-85.

27. Baroncelli GI, Federico G, Vignolo M, et al, 2006 Crosssectional reference data for phalangeal quantitative ultrasound from early childhood to young-adulthood according to gender, age, skeletal growth, and pubertal development. Bone 39: 159-173.

28. Cole TJ, Green PJ, 1992 Smoothing reference centile curves: the LMS method and penalized likelihood. Stat Med 11: 1305-1319.

29. Schoenau E, Saggese G, Peter F, et al, 2004 From bone biology to bone analysis. Horm Res 61: 257-269.

30. Kroger H, Kotaniemi A, Kroger L, Alhava E, 1993 Development of bone mass and bone density of the spine and femoral neck. A prospective study of 65 children and adolescents. Bone Miner 23: 171-182.

31. Cadossi R, Canè V, 1996 Pathways of transmission of ultrasound energy through the distal metaphysis of the second phalanx of pigs: an in vitro study. Osteoporos Int 6: 196-206.

32. Barkmann R, Rohrschneider W, Vierling M, et al, 2002 German paediatric reference data for quantitative transverse transmission ultrasound of finger phalanges. Osteoporos Int 13: 55-61.

33. Landin LA, 1983 Fracture patterns in children. Analysis of 8,682 fractures with special reference to incidence, etiology and secular changes in a Swedish urban population 1950-1979. Acta Orthop Scand Suppl 202: 1-109.

34. Bertelloni S, Baroncelli GI, Mora S, 2010 Bone health in disorders of sex differentiation. Sex Dev 4: 270-284.

35. Garden AS, Diver MJ, Fraser WD, 1996 Undiagnosed morbidity in adult women with Turner's syndrome. Clin Endocrinol (Oxf) 45: 589-593.

36. Gravholt CH, Vestergaard P, Hermann AP, Mosekilde L, Brixen K, Christiansen JS, 2003 Increased fracture rates in Turner's syndrome: a nationwide questionnaire survey. Clin Endocrinol (Oxf) 59: 89-96.

37. Han TS, Cadge B, Conway GS, 2006 Hearing impairment and low bone mineral density increase the risk of bone fractures in women with Turner's syndrome. Clin Endocrinol (Oxf) 65: 643-647.

38. Zuckerman-Levin N, Yaniv I, Schwartz T, Guttmann H, Hochberg Z, 2007 Normal DXA bone mineral density but frail cortical bone in Turner's syndrome. Clin Endocrinol (Oxf) 67: 60-64.

39. Bakalov VK, Axelrod L, Baron J, et al, 2003 Selective reduction in cortical bone mineral density in turner syndrome independent of ovarian hormone deficiency. J Clin Endocrinol Metab 88: 5717-5722.

40. Hansen S, Brixen K, Gravholt CH, 2012 Compromised trabecular microarchitecture and lower finite element estimates of radius and tibia bone strength in adults with turner syndrome: A cross-sectional study using high-resolution-pQCT. J Bone Miner Res 27: 1794-1803.

41. Baroncelli GI, 2008 Quantitative ultrasound methods to assess bone mineral status in children: technical characteristics, performance, and clinical application. 
Pediatr Res 63: 220-228.

42. Di Iorgi N, Calandra E, Secco A, et al, 2010 Quantitative ultrasound detects bone changes following bone marrow transplantation in pediatric subjects with hematological diseases: a longitudinal study. J Endocrinol Invest 33: 478-482.

43. Baroncelli GI, Vierucci F, Bertelloni S, Erba P, Zampollo E, Giuca MR, 2013 Pamidronate treatment stimulates the onset of recovery phase reducing fracture rate and skeletal deformities in patients with idiopathic juvenile osteoporosis: comparison with untreated patients. J Bone
Miner Metab 31: 533-543.

44. Mussa A, Bertorello N, Porta F, et al, 2010 Prospective bone ultrasound patterns during childhood acute lymphoblastic leukemia treatment. Bone 46: 1016-1020.

45. Zywiec J, Pluskiewicz W, Adamczyk P, Skubala A, Gumprecht J, 2012 Phalangeal quantitative ultrasound measurements in chronic hemodialysis patients: a 4-year follow-up. Ultrasound Med Biol 38: 962-971.

46. Mussa A, Porta F, Baldassarre G, et al, 2012 Phalangeal quantitative ultrasound in 1,719 children and adolescents with bone disorders. Osteoporos Int 23: 1987-1998. 